\title{
Quantum and classical ratchet motions of vortices in a two-dimensional trigonal superconductor
}

\author{
Yuki M. Itahashi $\odot,{ }^{1, *}$ Yu Saito, ${ }^{1,2, *}$ Toshiya Ideue $\odot,{ }^{1, \dagger}$ Tsutomu Nojima $\odot,{ }^{3}$ and Yoshihiro Iwasa ${ }^{1,4, \ddagger}$ \\ ${ }^{1}$ Quantum-Phase Electronics Center (QPEC) and Department of Applied Physics, The University of Tokyo, Tokyo 113-8656, Japan \\ ${ }^{2}$ California NanoSystems Institute, University of California at Santa Barbara, Santa Barbara, California 93106, USA \\ ${ }^{3}$ Institute for Materials Research, Tohoku University, Sendai 980-8577, Japan \\ ${ }^{4}$ RIKEN Center for Emergent Matter Science (CEMS), Wako 351-0198, Japan
}

(Received 25 November 2019; revised manuscript received 11 March 2020; accepted 7 April 2020; published 4 May 2020)

\begin{abstract}
Dynamical behavior of vortices plays central roles in the quantum phenomena of two-dimensional (2D) superconductors. The quantum metallic state, for example, showing an anomalous temperature-independent resistive state down to low temperatures, has been a topical subject in recently developed 2D crystalline superconductors, whose microscopic origin is still under debate. Here, we unveil an interesting aspect of the vortex dynamics in a noncentrosymmetric 2D crystalline superconductor of gated $\mathrm{MoS}_{2}$ through the nonreciprocal transport measurement. The second-harmonic resistance $R^{2 \omega}$ at low temperature with high current indicates the classical vortex flow accompanying the ratchet motion. Furthermore, we found that $R^{2 \omega}$ is substantially suppressed in the quantum metallic state with the low current region, allowing identification of the quantum and classical ratchet motions of vortices by the magnitude of the second-harmonic resistance. Combining $R^{2 \omega}$ with the linear resistance $R^{\omega}$ allowed us to establish a comprehensive vortex phase diagram for crystalline 2D superconductors with minimal disorder.
\end{abstract}

DOI: 10.1103/PhysRevResearch.2.023127

\section{INTRODUCTION}

A variety of two-dimensional (2D) superconductors have emerged in the past decade, providing a novel materials' platform of unique physical phenomena [1-5]. Among them, quantum phases and their transitions in the vortex states are of particular interest [6-9]. In conventional amorphous metallic films, a superconductor-insulator transition occurring at a single critical point in the zero-temperature limit is one of the well-known quantum phenomena $[10,11]$. Such quantum critical behavior is predominantly controlled by the disorder of films, which are inevitably enhanced by the reduction of film thickness, and hence well described by the scaling theory in the framework of the dirty boson picture [11].

On the other hand, a new route has been desired to approach the quantum phase transition with minimal disorder, for the comprehensive understanding of 2D superconductors. The recently emerging $2 \mathrm{D}$ superconductors based on single crystals $[1,2]$ are one of such candidate materials, and in fact, some of them reveal a completely different physical picture from the dirty boson model [10]. Instead of superconducting and insulating states separated by the quantum critical point,

\footnotetext{
${ }^{*}$ These authors contributed equally to this work.

†ideue@ap.t.u-tokyo.ac.jp

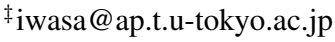

Published by the American Physical Society under the terms of the Creative Commons Attribution 4.0 International license. Further distribution of this work must maintain attribution to the author(s) and the published article's title, journal citation, and DOI. gate-induced and exfoliated 2D superconductors display a broad metallic state dominating a magnetic field and temperature $(B-T)$ phase diagram [6,7]. In this state, once the out-ofplane magnetic field is applied, the zero-resistance state is immediately destroyed into the temperature-independent finite resistive state even at low temperatures. Such a resistive state is observed not only in the $2 \mathrm{D}$ crystalline superconductors but also in amorphous thin film superconductors with weak pinning effect $[8,12,13]$, though its origin is still under debate. In addition to the extrinsic heating by the environmental noise [14], several intrinsic mechanisms such as quantum collective creep of vortices [12,15] and Bose metal [7,16,17] are being discussed. However, it is technically difficult to distinguish such various vortex states by direct microscopic observation, especially in nanometer-thick flake crystals, which hinders nontransport probes.

As an alternative probe of vortex states, we introduce, in this study, nonreciprocal phenomena, which are sensitively probed by the second-harmonic magnetoresistance. It is generally known that, in a noncentrosymmetric system, the electrical resistance becomes dependent on the current direction, when the time reversal symmetry is broken [18]. Although nonreciprocal transport was originally studied in artificial helical structures or interfaces [18,19], it is nowadays applied to various noncentrosymmetric crystalline systems $[20,21]$, including superconductors without inversion symmetry [22-24]. Among them, ion-gated $\mathrm{MoS}_{2}$, one of the widely studied transition metal dichalcogenides [25,26], is considered to be a trigonal 2D superconductor [Fig. 1(a)], and thus can be an ideal platform for investigating the vortex dynamics through nonreciprocal transport. 
(a)

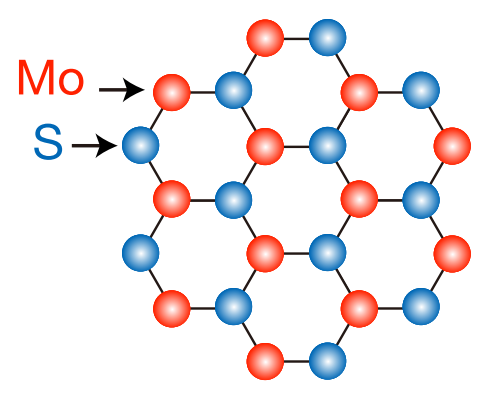

(b)

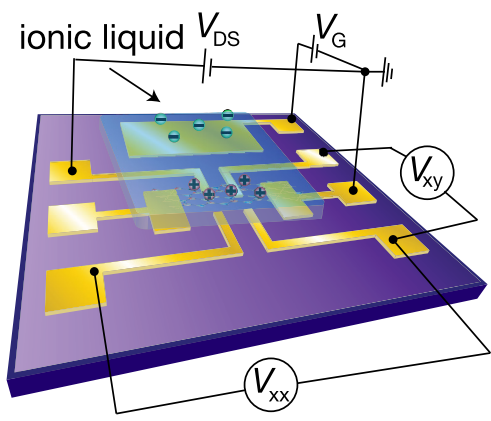

(c)

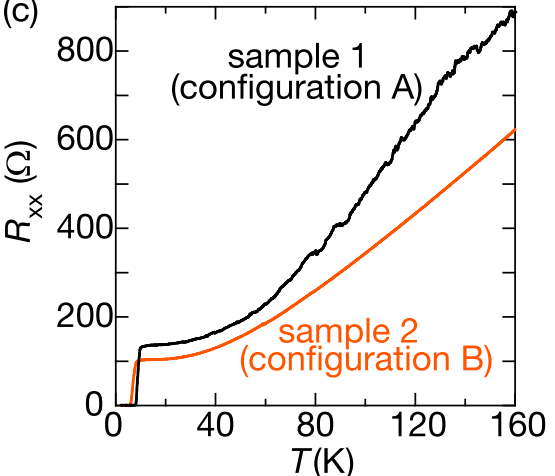

(d)

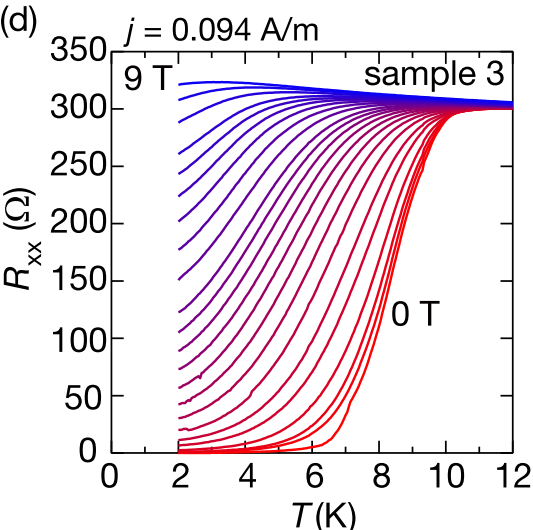

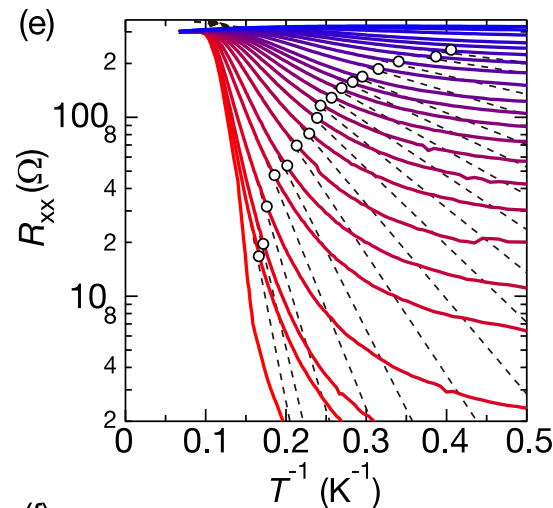

(f)

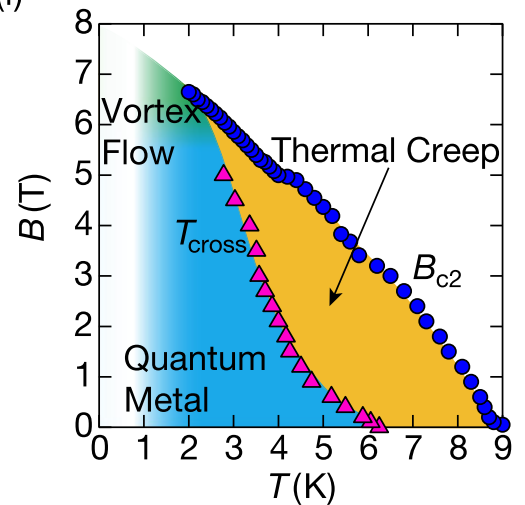

FIG. 1. Gate-induced superconductivity and vortex phase diagram in $\mathrm{MoS}_{2}$. (a) Top view of trigonal crystal structure of MoS 2 . (b) Schematic image of EDLT. (c) $R_{x x}$ as a function of $T$ in samples 1 (black line) and 2 (orange line) for the magnetic field at 0 T. Samples 1 and 2 correspond to configurations $\mathrm{A}$ and $\mathrm{B}$, respectively. (d) $R_{x x}$ as a function of $T$ in sample 3 under various perpendicular magnetic fields at a low current of $0.94 \mathrm{~A} / \mathrm{m}$. Sample 3 corresponds to configuration A. The magnetic field was varied in 0.05-T steps from 0 to $0.1 \mathrm{~T}$, in $0.2-\mathrm{T}$ steps from 0.2 to $0.6 \mathrm{~T}$, in 0.3-T steps from 0.9 to $3 \mathrm{~T}$, in 0.5-T steps from 3.5 to $6 \mathrm{~T}$, and in 1-T steps from 7 to $9 \mathrm{~T}$. (e) Arrhenius plot of $R_{x x}$ in sample 3 for the same data as the ones in (d). The black dashed lines show the activated behavior of vortices described as $R_{x x} \propto \exp \left(-\frac{U}{k_{\mathrm{B}} T}\right)$. White circles show boundary between the thermal creep and quantum creep regimes $\left(T_{\text {cross }}\right) . T_{\text {cross }}$ is determined as temperatures at which $R_{x x}$ deviate from the activated behavior. (f) Vortex phase diagram in $\operatorname{MoS}_{2} \operatorname{EDLT}$ at $j=0.94 \mathrm{~A} / \mathrm{m}$. The upper critical field curve $B_{\mathrm{c} 2}(T)$ or $T_{\mathrm{c}}(B)$ (blue circles) is defined as $B$ or $T$ with $90 \%$ of the normal state resistance and $T_{\text {cross }}$ (pink triangles) is determined from the Arrhenius plot as shown by the white circles in (e). Orange and light blue regions show thermal creep and quantum metal region, respectively.

Here, we report a study on nonreciprocal charge transport, which sensitively probes the vortex ratchet motion reflecting lattice symmetry, in ion-gated $\mathrm{MoS}_{2}$ single crystals. We found that the anisotropic behavior in the secondharmonic resistance satisfies the selection rules for threefold symmetry of single-layer $\mathrm{MoS}_{2}$, which indicates that the gateinduced superconductivity in multilayer $\mathrm{MoS}_{2}$ obeys the symmetry of the monolayer. More importantly, the nonreciprocal magnetoresistance observed down to the lowest temperature at a relatively high current density can be understood in terms of not the paraconductivity scenario near $T_{\mathrm{c}}$ [23] but the ratchet motion of vortices in the plastic flow regime, which is caused by the asymmetric restraining force with threefold symmetry. Furthermore, we found that the second-harmonic resistance signal at a low current is substantially suppressed below a certain magnetic field, being consistent with the quantum creep (tunneling) picture of vortices in noncentrosymmetric media. The present result indicates that nonreciprocal transport serves as an effective probe to differentiate the quantum and classical nature of vortex dynamics in noncentrosymmetric superconductors.

\section{RESULTS}

\section{A. Transport properties of $\mathrm{MoS}_{2}$ EDLT}

We prepared three electric double-layer transistor (EDLT) samples [Fig. 1(b); see Appendix A] with different configurations using two different current directions: parallel to the zigzag direction (configuration A; samples 1 and 3 ) and parallel to the armchair direction (configuration B; sample 2), as shown in Fig. 2(a). Carrier densities of samples 1, 2, and 3 are $1.2 \times 10^{14} \mathrm{~cm}^{-2}, 1.8 \times 10^{14} \mathrm{~cm}^{-2}$, and $1.3 \times 10^{14} \mathrm{~cm}^{-2}$, respectively, which were estimated by the Hall resistance measurements at $15 \mathrm{~K}$. The crystal orientations of the $\mathrm{MoS}_{2}$ flakes were determined from the shape of the edge according to a previous study [27] (see Appendix A).

We then performed the ac transport measurements (see Appendix A). First, we measured the first $\left(R^{\omega}\right)$ harmonic signals of longitudinal resistance $\left(R_{x x}\right)$ to show typical superconducting properties in the present system. Figure 1(c) shows $R_{x x}$ as a function of temperature $T$ for sample 1 (black) and sample 2 (orange) in zero magnetic field at a gate voltage of $V_{\mathrm{G}}=5 \mathrm{~V}$. As $T$ decreases, $R_{x x}$ continuously decreases and suddenly goes 

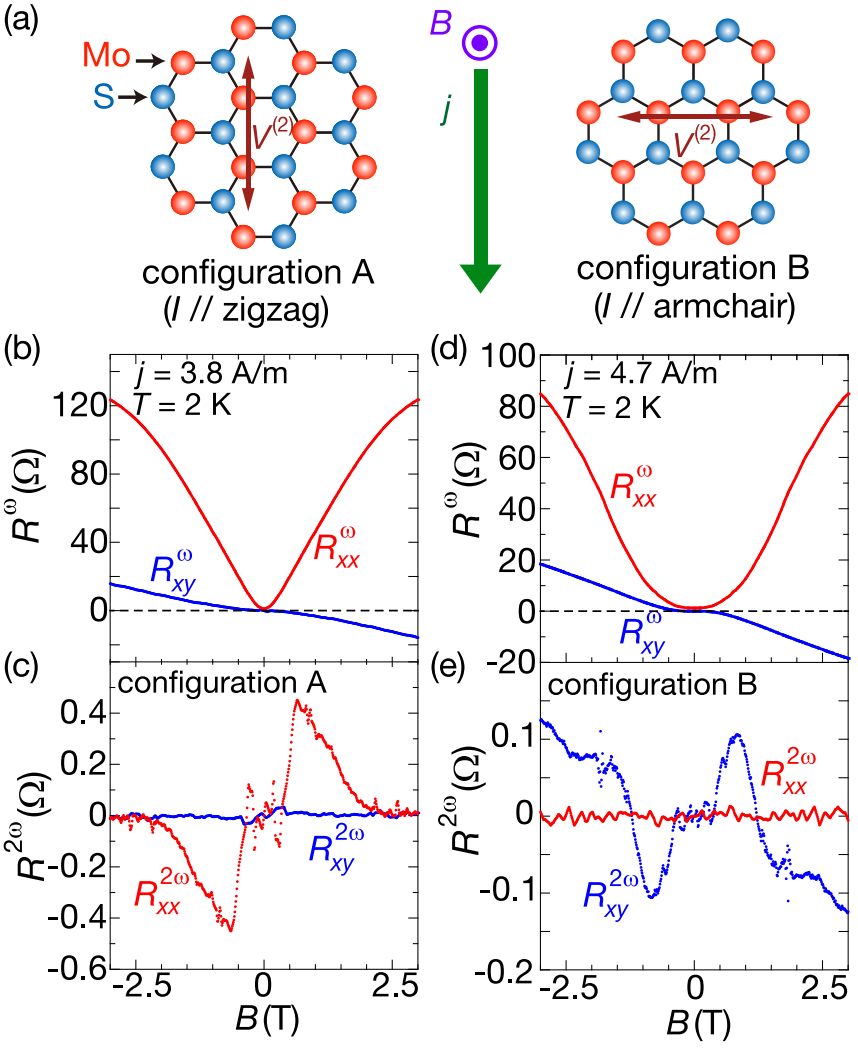

FIG. 2. Selection rules of nonreciprocal transport reflecting threefold symmetry. (a) Two kinds of device configurations depending on the crystal orientation of $\mathrm{MoS}_{2}$. In configuration A (B), the applied current is parallel to the zigzag (armchair) direction, and a second-harmonic signal of the longitudinal (transverse) component is expected (see Appendix C for details). (b), (c) Longitudinal $\left(R_{x x}\right.$ : red) and transverse $\left(R_{x y}\right.$ : blue) (b) first $\left(R^{\omega}\right)$ and (c) second $\left(R^{2 \omega}\right)$ harmonic magnetoresistance when $T=2 \mathrm{~K}$ and $j=3.8 \mathrm{~A} / \mathrm{m}$ in configuration $\mathrm{A}$, which is shown in the inset. $R_{x x}^{\omega}$ is symmetrized and others are antisymmetrized (see Appendix B for details). (d), (e) Longitudinal $\left(R_{x x}:\right.$ red) and transverse $\left(R_{x y}\right.$ : blue); (d) first- $\left(R^{\omega}\right)$ and (e) second$\left(R^{2 \omega}\right)$ harmonic magnetoresistance when $T=2 \mathrm{~K}$ and $j=4.7 \mathrm{~A} / \mathrm{m}$ in configuration $\mathrm{B}$, which is shown in the inset. $R_{x x}^{\omega}$ is symmetrized and others are antisymmetrized.

to zero below $10 \mathrm{~K}$. The $T_{\mathrm{c}}$ of samples 1 and 2, defined as $90 \%$ of the resistive transitions, are 9.6 and $7.3 \mathrm{~K}$, respectively. Figure 1(d) shows $R_{x x}-T$ in sample 3 with $T_{\mathrm{c}}=9.6 \mathrm{~K}$ under various magnetic fields $B(0-9 \mathrm{~T})$ at a source-drain linear current density $(j)$ of $0.094 \mathrm{~A} / \mathrm{m}$. The linear current density in two-dimensional systems is calculated as $j=I / W$, where $I$ and $W$ are the source-drain current and the sample width, respectively (in samples 1,2 , and $3, W=3.4,3.2$, and $5.3 \mu \mathrm{m}$, respectively). The drop of sheet resistance becomes broadened with increasing $B$, and the transition disappears near $9 \mathrm{~T}$. Figure 1(e) shows an Arrhenius plot of $R_{x x}$ in sample 3 [the same data as those in Fig. 1(d)]. At high temperatures near $T_{\mathrm{c}}$, the data show activated behavior indicating the thermal creep region $[6,28,29]$, while at lower temperatures, the resistance deviates from the thermally activated behavior and shows the almost temperature-independent behavior. Figure 1(f) shows a vortex phase diagram based on the analyses in Fig. 1(e) for sample 3, where the upper critical field curve $B_{\mathrm{c} 2}(T)$ is derived from $B$ and $T$ with $90 \%$ of normal state resistance, which is confirmed to be close to the mean field value [28]. Below $T_{\text {cross }}$, which is determined as temperatures at which $R_{x x}$ deviates from the activated behavior [white circles in Fig. 1(e)], the phase diagram is dominated by the quantum metallic state, in agreement with the previous study [28].

\section{B. Nonreciprocal transport}

Next, we focus on the second $\left(R^{2 \omega}\right)$ harmonic signals of longitudinal $\left(R_{x x}\right)$ and transverse $\left(R_{x y}\right)$ resistance. Figures 2(b)-2(e) show the first and second harmonic signals as a function of $B$, at $T=2 \mathrm{~K}$ and $j=3.8 \mathrm{~A} / \mathrm{m}(4.7 \mathrm{~A} / \mathrm{m})$ in configuration A (B). As $B$ increases, the $R^{2 \omega}$ signal $\left[R_{x x}\right.$ in Fig. 2(c) and $R_{x y}$ in Fig. 2(e)] observed in both configurations first increases, then reaches a peak and finally decreases to almost zero. This peak behavior of $R^{2 \omega}$ in the $B$ region with small absolute values of $R^{\omega}$ indicates that the nonreciprocal signals are enhanced in the superconducting state, as shown in the previous study [23]. In addition, we note that in the configuration A (B), only the $R^{2 \omega}$ component of $R_{x x}\left(R_{x y}\right)$ is observed without a discernible signal in the other component. The $B$-linear background of the $R^{2 \omega}$ component observed in sample 2 [Fig. 2(e)] is possibly coming from the extrinsic origin due to the second-harmonic component of $j$, which inevitably appears because of geometrical unbalances in the source-drain electrodes (see Appendix B). This directionaldependent presence or absence of the second-harmonic signals is consistent with the threefold symmetry of the crystal structure, reflecting the high crystallinity of the present 2D superconducting system (see Appendix C). Also, it is stressed that the inversion symmetry in multilayer $2 \mathrm{H}-\mathrm{MoS}_{2}$ is broken in the gated $\mathrm{MoS}_{2}$, in a manner analogous to the monolayer $\mathrm{MoS}_{2}$. The observation of reduced symmetry by the gate electric field is consistent with the consideration in the previous paper [30,31].

Figures 3(a) and 3(b) show the $R^{\omega}$ and $R^{2 \omega}$ components of $R_{x x}\left(R_{x x}^{\omega}\right.$ and $\left.R_{x x}^{2 \omega}\right)$, respectively, as a function of $B$ at various temperatures $(2-10 \mathrm{~K})$ in sample 1 with the configuration A for $j=3.8 \mathrm{~A} / \mathrm{m}$. We found that the amplitude of $R_{x x}^{2 \omega}$ at the peak position $\left(R_{\text {peak }}^{2 \omega}\right)$ increases with decreasing temperature [Fig. 3(c)] and is indiscernible in $T>T_{\mathrm{c}}$. In the previous study [23], our argument was only near $T_{\mathrm{c}}$, where the transport is well described in terms of paraconductivity. However, at temperatures well below $T_{\mathrm{c}}$, which is our main focus in this work, the vortex dynamics plays a crucial role in the enhancement of nonreciprocal transport, because the transport far below $T_{\mathrm{c}}$ and $B_{\mathrm{c} 2}$ is governed by vortex motion.

In order to elucidate the origin of nonreciprocal transport at lower temperatures far below $T_{\mathrm{c}}$, we measured the $B$ dependence of $R_{x x}^{\omega}$ and $R_{x x}^{2 \omega}$ [Figs. 3(d) and 3(e)] for various values of $j(1.5-13 \mathrm{~A} / \mathrm{m})$ at $2 \mathrm{~K}$ in sample 1. As shown in Figs. 3(d) and $3(\mathrm{e})$, in the low current region $(j=1.5-5.3 \mathrm{~A} / \mathrm{m})$, there exists a region of $B$ where $R_{x x}^{\omega}$ is finite while $R_{x x}^{2 \omega}$ is indiscernible below a certain magnetic field $B_{1}$ (white triangle). Above $B_{1}, R_{x x}^{2 \omega}$ develops, showing a peak structure, and then decreases toward the second threshold magnetic field $B_{2}$ (black triangle). Above $B_{2}, R_{x x}^{2 \omega}$ becomes very small. As $j$ 

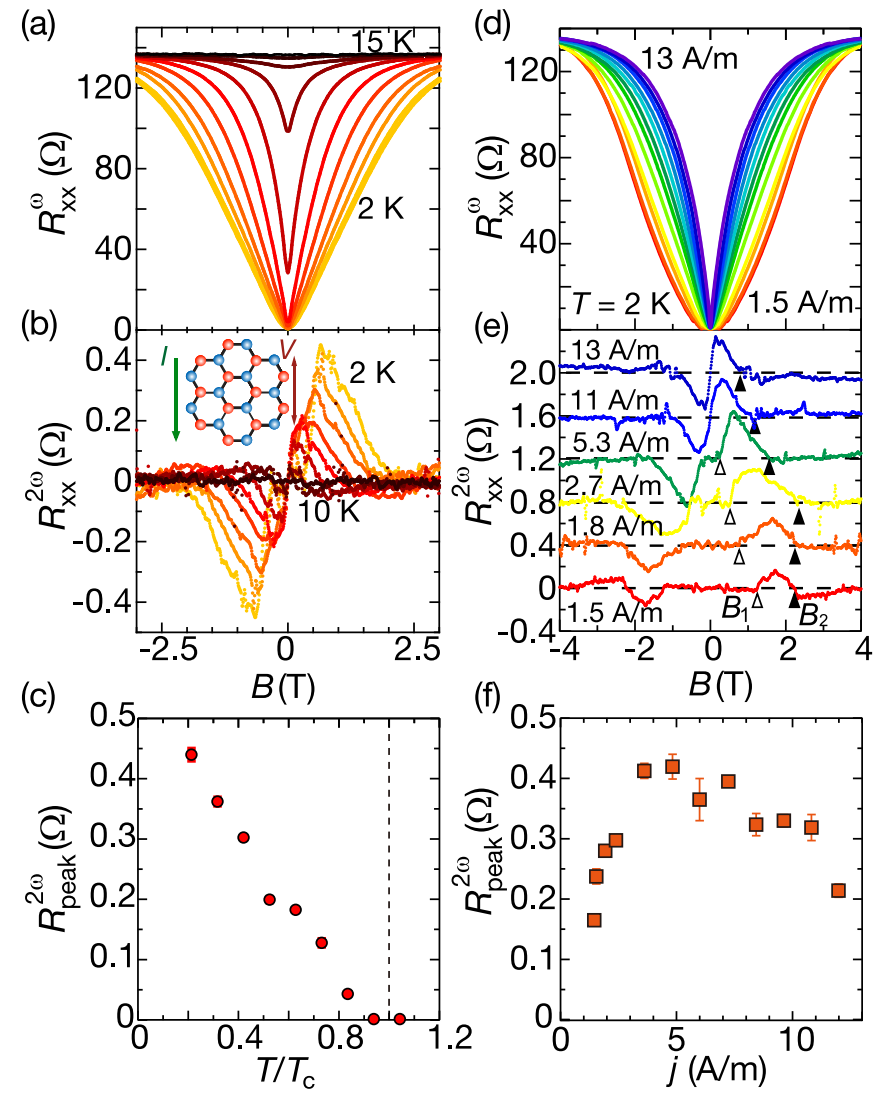

FIG. 3. First- and second-harmonic magnetoresistance at various temperatures and currents for the longitudinal direction. (a) Longitudinal first-harmonic magnetoresistance $\left(R_{x x}^{\omega}\right)$ for temperatures varying in $1-\mathrm{K}$ steps from 2 to 10,12 , and $15 \mathrm{~K}$ for $j=3.8 \mathrm{~A} / \mathrm{m}$ in configuration $\mathrm{A}$, which is shown in the inset of (b). (b) Longitudinal second-harmonic magnetoresistance $\left(R_{x x}^{2 \omega}\right)$ for temperatures varying in $1-\mathrm{K}$ steps from 2 to $10 \mathrm{~K}$ for $j=3.8 \mathrm{~A} / \mathrm{m}$. $R_{x x}^{\omega}$ is symmetrized and $R_{x x}^{2 \omega}$ is antisymmetrized. (c) Amplitude of second-harmonic resistance at the peak position $\left(R_{\text {peak }}^{2 \omega}\right)$ extracted from (b), as a function of temperature normalized with $T_{\mathrm{c}}\left(T / T_{\mathrm{c}}\right)$. (d) Longitudinal magnetoresistance $\left(R_{x x}^{\omega}\right)$ for currents at 1.5, 1.8, 2.1, 2.7, 3.8, 5.3, $6.8,8.2,9.4,11,12$, and $13 \mathrm{~A} / \mathrm{m}$ at $T=2 \mathrm{~K}$ in configuration A. (e) Second-harmonic magnetoresistance $\left(R_{x x}^{2 \omega}\right)$ for currents at 1.5, 1.8, $2.7,5.3,11$, and $13 \mathrm{~A} / \mathrm{m}$. Each curve is shifted vertically by 0.4 $\Omega$ for clarity. $R_{x x}^{\omega}$ is symmetrized and $R_{x x}^{2 \omega}$ is antisymmetrized. $R_{x x}^{2 \omega}$ is substantially enhanced between $B_{1}$ and $B_{2}$, as indicated by white and black triangles, respectively. (f) Amplitude of $R_{x x}^{2 \omega}$ at the peak position $\left(R_{\text {peak }}^{2 \omega}\right)$ as a function of $j$ extracted from $R_{x x}^{2 \omega}$ data. Error bars indicate uncertainty of $R_{\text {peak }}^{2 \omega}$.

increases, $B_{1}$ gradually decreases and becomes almost $0 \mathrm{~T}$ at the higher current region $(j<5.3 \mathrm{~A} / \mathrm{m})$. Figure 3(f) displays $R_{\text {peak }}^{2 \omega}$ as a function of $j$. The characteristic domelike behavior is similar to that of the vortex ratchet effect observed in thin films with artificial asymmetric potentials [32-34], where the rectified ac-driven vortices produce a dc electric field showing a domelike dependence on the ac current. The decrease of the rectification effect is due to the effective weakening of the pinning potentials by increasing the ac current.

According to a theoretical paper, nonreciprocal transport occurs when the vortices move among the pinning potentials as classical particles, in two-dimensional noncentrosymmetric superconductors [35]. Since the possible defects in $\mathrm{MoS}_{2}$ mono and few layers are the sulfur vacancies, they likely play important roles as pinning centers of $2 \mathrm{D}$ superconductivity. Considering that the length scale of the sulfur vacancies is much smaller than that of the vortex core, the collective pinning rather than individual ones may be the plausible picture describing our system. Namely, a limited number of weak pinning centers ( $\mathrm{S}$ vacancies) collectively pin the vortex lattices. Thus, the pinning potential here can have a larger scale than that of the single pinning center. The motion of vortex lattices is driven by the Lorentz force from the supercurrent. According to Hoshino et al. [35], the superfluid density is directionally dependent owing to the broken inversion symmetry. Therefore, the condensation energy at the pinning center becomes dependent on the current direction, resulting in the asymmetry in the collective pinning potential. The vortex ratchet effect may naturally appear as a consequence of disorders in a noncentrosymmetric system.

For a further understanding of the relation between vortex motion and nonreciprocal resistance at the low current region, we plot $R_{x x}^{2 \omega}$ and $R_{x x}^{\omega}$ at $j=1.5 \mathrm{~A} / \mathrm{m}$ against $B$ in Figs. 4(a) and 4(b), respectively. As mentioned in Fig. 3(e), $R_{x x}^{2 \omega}$ appears at $B_{1}$ (white triangle) and is suppressed at $B_{2}$ (black triangle). Figure 4(b) shows that, in this particular device, the zeroresistance (ZR) state is observed at finite magnetic field up to $0.25 \mathrm{~T}$ (orange triangle), above which $R_{x x}^{\omega}$ increases in a nonlinear manner with $B$. Above $1.25 \mathrm{~T}, R_{x x}^{\omega}$ becomes quasi$B$-linear. According to Ref. [6], the region of $0.25 \mathrm{~T}<B<$ 1.25 $\mathrm{T}$ and $1.25 \mathrm{~T}<B$ are assigned as the quantum metallic and the vortex flow states, respectively. Importantly, the onset magnetic field $B_{1}$ of $R_{x x}^{2 \omega}$ well agrees with the crossover field of $1.25 \mathrm{~T}$. This result indicates that $R_{x x}^{2 \omega}$; in other words, the vortex ratchet effect is small in the quantum metallic region, whereas it is dramatically enhanced in the vortex flow region.

In the plastic vortex flow region above $B_{1}$, the weakly pinned vortices move with the thermally activated process and their motions should be affected by the asymmetric pinning potentials reflecting the trigonal lattice symmetry. Therefore the vortex motion in this state is regarded as a classical ratchet. On the other hand, in the quantum metallic region, the vortices hop between the local potential minima through the tunneling process (more strictly, quantum collective creep), which is predominantly determined by the wave-function overlap. In such a quantum mechanical process with wave nature, the vortex motion might be less dependent on the directions. The suppression of the ratchet effect in quantum mechanical motion and the enhanced ratchet effect in classical motions is consistent with the general physical understanding of the ratchet effect, which has been theoretically discussed [36,37]. The above considerations indicate that crossover from the quantum to classical ratchets, in other words, from the quantum metallic state to the vortex flow state, can be unambiguously determined from $R_{x x}^{2 \omega}$.

With increasing magnetic fields, the nonreciprocal signal $R_{x x}^{2 \omega}$ is again suppressed above $B_{2}$, which is close to the magnetic field where $R_{x x}^{\omega}$ becomes half of the normal state value. In this region, the picture of the plastic vortex flow does not hold due to the weakening of pinning potentials. Then the vortices flow rather freely without the ratchet effect, leading 

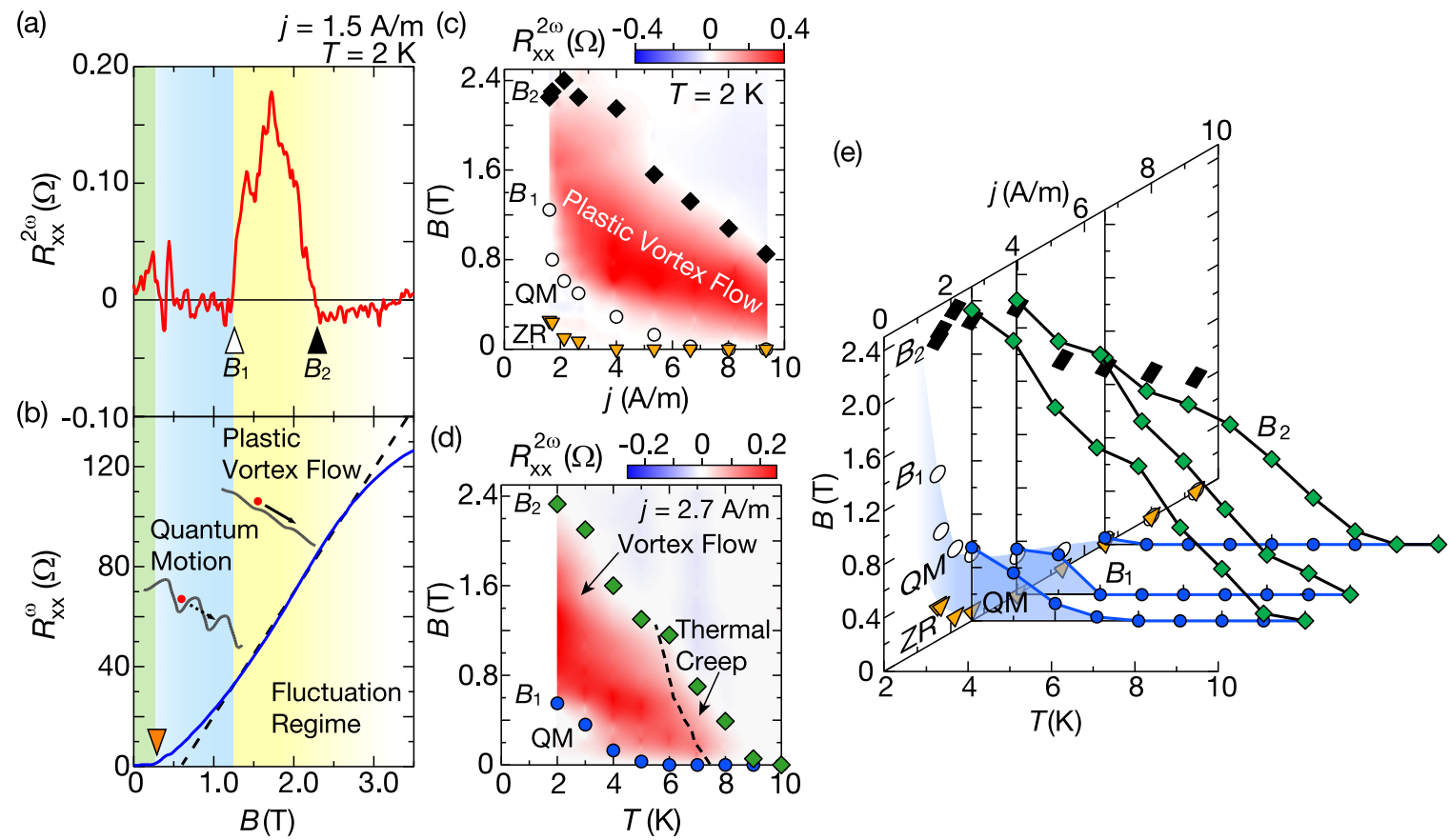

FIG. 4. Second- and first-harmonic magnetoresistance and vortex phase diagram. (a,b) Magnetic field dependence of $R_{x x}^{2 \omega}$ (a) and $R_{x x}^{\omega}$ (b) at $T=2 \mathrm{~K}, j=1.5 \mathrm{~A} / \mathrm{m} . R_{x x}^{2 \omega}$ is significantly enhanced between $B_{1}$ and $B_{2}$ indicated by white and black triangles, respectively. This particular sample shows the zero $R_{x x}^{\omega}$. The zero-resistance (ZR) state exists up to the magnetic field indicated by the orange triangle. The black dashed line shows the $B$-linear dependence of $R_{x x}^{\omega}$ above $1.25 \mathrm{~T}$. The green, blue, yellow, and white backgrounds indicate the magnetic field regions, which are separated by the three triangles. Inset schematics display conceptual images of vortex motion in the corresponding magnetic field regions (see main text). (c) A dynamical vortex diagram in the $B$ - $j$ plane drawn by $R_{x x}^{2 \omega}$. White circles and black diamonds represent $B_{1}$ and $B_{2}$ extracted from (a) and Fig. 3(e). Orange triangles show the magnetic field, where the ZR state disappears as extracted from the $R_{x x}^{\omega}-B$ curves for various values of $j$. Color mapping displays the magnitude of $R_{x x}^{2 \omega}$ in the region of $0 \mathrm{~T}<B<2.5 \mathrm{~T}$ and $1.5 \mathrm{~A} / \mathrm{m}<j<9.4 \mathrm{~A} / \mathrm{m}$. (d) $B-T$ vortex phase diagram at $j=2.7 \mathrm{~A} / \mathrm{m}$ obtained from $R_{x x}^{2 \omega}$. Blue circles and green triangles indicate $B_{1}$ and $B_{2}$, respectively. Color mapping displays the magnitude of $R_{x x}^{2 \omega}$ in the region of $0 \mathrm{~T}<B<2.5 \mathrm{~T}$ and $2 \mathrm{~K}<T<10 \mathrm{~K}$. The quantum metal (QM) region is unambiguously identified by measuring $R_{x x}^{2 \omega}$. The black dashed line in (d) is the boundary between the vortex flow and the thermal creep states extracted from $R_{x x}^{\omega}$ in a similar manner to Fig. 1(e), providing a complete vortex phase diagram at the low current density. (e) A comprehensive $B$-j- $T$ phase diagram for vortex dynamics. Light blue regions indicate the quantum metallic state.

to the suppression of $R_{x x}^{2 \omega}$. It is noted that this free vortex flow region continuously connects to the quantum amplitude fluctuation region [28].

\section{DISCUSSION}

In Fig. 4(c), we provide a dynamical phase diagram at $T=2 \mathrm{~K}$ obtained from the nonreciprocal signals. Both the zero-resistance (ZR) state and quantum metallic (QM) states are quickly suppressed by the current, and the plastic vortex flow region becomes dominant. With further increasing $j$, the plastic flow region is also suppressed, because superconductivity is almost killed by the current. The strong red color indicates a large $R_{x x}^{2 \omega}$ signal from the classical ratchet effect in the plastic vortex flow region, which takes a crucial role in determining this dynamical vortex phase diagram.

Figure 4(d) represents the $B-T$ vortex phase diagram at $j=2.7 \mathrm{~A} / \mathrm{m}$ drawn by the magnitude of $R_{x x}^{2 \omega}$. $B_{1}$ (blue circles) and $B_{2}$ (green diamonds) are determined from the temperature variations of $R_{x x}^{2 \omega}-B$ curves in a similar manner to Figs. 3(e) or 4(a) (see Appendix D). It is noted that the nonlinear response $R_{x x}^{2 \omega}$ enables us to clearly distinguish the quantum metallic state and the vortex flow state at the lowest temperature, which was difficult in the linear response $R_{x x}^{\omega}$. The black dashed line in Fig. 4(d) is the boundary of the vortex flow and the thermal creep states extracted from $R_{x x}^{\omega}$ in a similar manner to Fig. 1(e), providing a complete vortex phase diagram at the low current density regime. A comprehensive $B-j-T$ vortex phase diagram from the $R_{x x}^{2 \omega}$ measurement is displayed in Fig. 4(e), which consists of the $B-T$ phase diagram observed at several $j$ values (see Appendix D). The plastic vortex flow region between $B_{1}$ and $B_{2}$ is smoothly connected to the dynamical $B-j$ plane at the lowest temperature.

We note that the sample temperature in the high current regions might be increased more or less in Fig. 4(c). However, the plastic vortex flow region does not seem to shrink abruptly with increasing current in our measurement range, implying the stable vortex dynamic state without quenching to the normal state. In addition, we observed the zero resistance in zero magnetic field at least until $13 \mathrm{~A} / \mathrm{m}$ as shown in Fig. 3(d). In comparison with the data of temperature dependence shown in Fig. 3(a), $R_{x x}^{\omega}(j=3.8 \mathrm{~A} / \mathrm{m}, B=0 \mathrm{~T})$ at $3 \mathrm{~K}$ is comparable to $R_{x x}^{\omega}(j=13 \mathrm{~A} / \mathrm{m}, B=0 \mathrm{~T})$ at $2 \mathrm{~K}$. Thus, the rough estimation of increase in the effective electron temperature by increasing current density of $10 \mathrm{~A} / \mathrm{m}$ is below $1 \mathrm{~K}$. These results imply 
that in our measurement range, the heating effect on the nonreciprocal signal is not so significant if it exists and the dynamical phase diagram is not qualitatively altered.

The suppression of nonreciprocal signals in the low current, magnetic field, and temperature regions supports the scenario of quantum tunneling of vortices for the quantum metallic state [6], contrary to the recent study [14], which attributes a quantum metallic state to the heating effect by the extrinsic noise. If the temperature-independent metallic state is induced by the heating effect from noise [14], this leads to the classical vortex motion, such as vortex flow or thermal creep, both of which should exhibit a large secondharmonic signal $R_{x x}^{2 \omega}$. This is contrary to the experimental results [Fig. 4(e)], where $R_{x x}^{2 \omega}$ is significantly suppressed. Therefore, we believe that the heating effect from noise can be excluded.

\section{CONCLUSION}

We reported a nonreciprocal charge transport study on ion-gated 2D superconductivity on $\mathrm{MoS}_{2}$ single crystals. The anisotropic behavior in the second-harmonic resistance satisfies the selection rules for threefold symmetry of single-layer $\mathrm{MoS}_{2}$, indicating that the gate-induced superconductivity in multilayer $\mathrm{MoS}_{2}$ obeys the symmetry of the monolayer. More importantly, we have found that the second-harmonic signal is dominated by the ratchet motion of vortices in the plastic flow regime, which is caused by the asymmetric restraining force with threefold symmetry. In sharp contrast, the secondharmonic resistance signal at a low current is substantially suppressed below a certain magnetic field in the quantum metallic state, where the quantum creep (tunneling) of vortices is playing a dominant role. Combining the linear and the nonreciprocal responses, we succeeded in establishing a dynamical phase diagram of vortices of 2D superconductors. The present result indicates that the nonreciprocal transport serves as a powerful tool to investigate the vortex dynamics in noncentrosymmetric superconductors.

\section{ACKNOWLEDGMENTS}

We thank N. Nagaosa, S. Hoshino, R. Wakatsuki, K. Hamamoto, H. Ueki, M. Ohuchi, J. Laurienzo, and Y. Nagai for fruitful discussions. Y.M.I. was supported by the Advanced Leading Graduate Course for Photon Science (ALPS). Y.S. was supported by the Japan Society for the Promotion of Science (JSPS) through a research fellowship for young scientists (Grant-in-Aid for JSPS Research Fellow, JSPS KAKENHI Grant No. JP15J07681). T.I. was supported by JSPS KAKENHI Grants No. JP19K21843, No. JP19H01819, and No. JP18H04216; the JST PRESTO project (Grant No. JPMJPR19L1); and a grant from the Yazaki Memorial Foundation for Science and Technology. T.N. was supported by JSPS KAKENHI Grant No. JP16H01061 (J-Physics). This work was supported by a Grant-in-Aid for Scientific Research (S) (JSPS KAKENHI Grant No. JP19H05602) from JSPS.

Y.S., T.I., and Y.I. conceived the research project. Y.S. designed the experiments. Y.M.I. fabricated samples. Y.M.I. and Y.S. performed the experiments and analyzed the data. All authors led the physical discussions and wrote the manuscript.
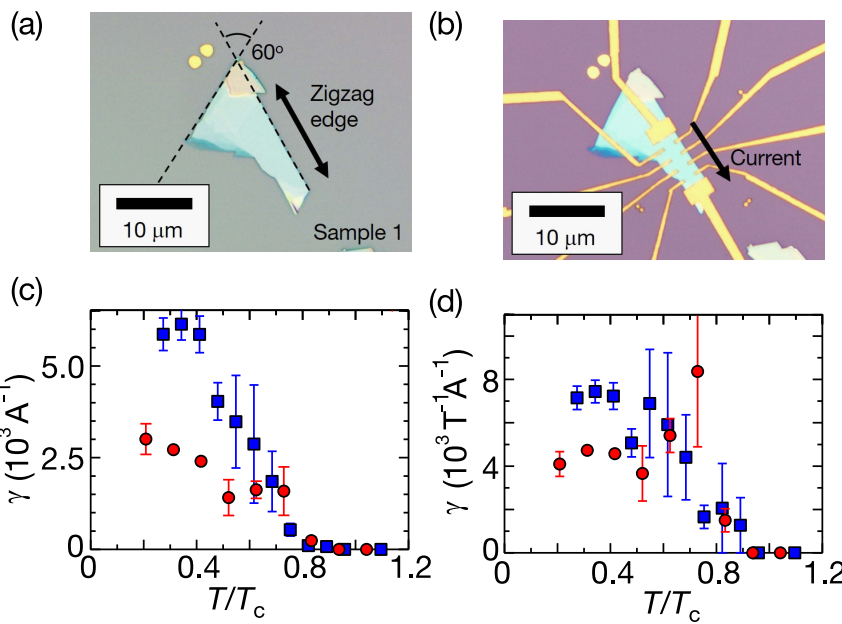

FIG. 5. Exfoliated $\mathrm{MoS}_{2}$ flake and comparison of nonreciprocal coefficients. (a) An optical image of the exfoliated $\mathrm{MoS}_{2}$ flake (sample 1). A straight edge is regarded as a zigzag edge. The angle between straight lines is $60^{\circ}$. (b) An optical image of the $\mathrm{MoS}_{2}$ device in configuration A (sample 1). Applied current is parallel to the zigzag edge. (c) $\gamma^{\prime}$ as a function of $T / T_{\mathrm{c}} \cdot \gamma^{\prime}$ is defined in Eq. (A2). (d) $\gamma$ as a function of $T / T_{\mathrm{c}} \cdot \gamma$ is defined in Eq. (A1), which is used in previous studies. Red circles and blue squares represent $\gamma$ and $\gamma^{\prime}$ calculated from $R_{x x}^{2 \omega}$ and $R_{x y}^{2 \omega}$, respectively.

\section{APPENDIX A: DEVICE FABRICATION AND TRANSPORT MEASUREMENTS}

Bulk $2 \mathrm{H}-\mathrm{MoS}_{2}$ single crystals were exfoliated into thin flakes by the Scotch-tape method and the flakes were transferred onto a $\mathrm{Si} / \mathrm{SiO}_{2}$ substrate. Thickness of exfoliated flakes were estimated to be $20 \mathrm{~nm}$ judging from optical contrasts. Also, steps which influence vortex motion were not found on the surface of the flakes. Hall bar configuration was fabricated onto the flakes with $\mathrm{Au}(90 \mathrm{~nm}) / \mathrm{Cr}(5 \mathrm{~nm})$ electrodes. The pattern was fabricated with electron beam lithography and electrodes were deposited with an evaporator. According to the previous study [27], straight edges in exfoliated $\mathrm{MoS}_{2}$ flakes correspond to zigzag edges with high probability. Thus, we selected $\mathrm{MoS}_{2}$ flakes with straight edges and judged the direction of flakes from their optical images [Figs. 5(a) and 5(b)]. After the deposition of electrodes, we put a droplet of ionic liquid on the surface of the $\mathrm{MoS}_{2}$ flake. We applied the gate voltage through the ionic liquid and realized a gate-induced 2D superconductivity. $\mathrm{N}, \mathrm{N}$-diethyl- $\mathrm{N}$ (2-methoxyethyl)- $N$ - methylammonium bis (trifluoromethylsulphonyl) imide (DEME-TFSI) was used as a gate medium.

The temperature-dependent resistance under a magnetic field was measured with standard four-probe geometry in a Quantum Design Physical Property Measurement System (PPMS) combined with two kinds of ac lock-in amplifiers (Stanford Research Systems Model SR830 DSP and Signal Recovery Model 5210) with a frequency of $13 \mathrm{~Hz}$. The gate voltage was supplied by a Keithley 2400 source meter at $220 \mathrm{~K}$, which is just above the glass transition temperature of DEME-TFSI, under high vacuum (less than $10^{-4}$ Torr). Below, we explain the details of the ac lock-in measurement technique. 
(a)

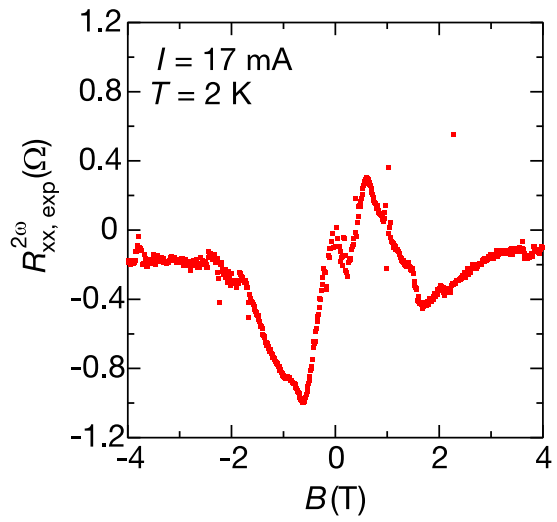

(b)

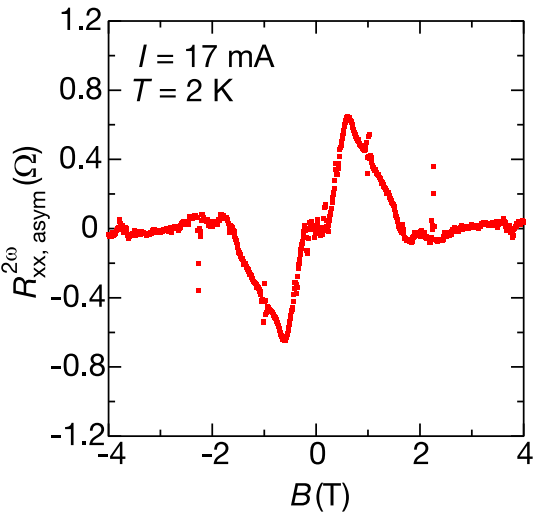

(c)

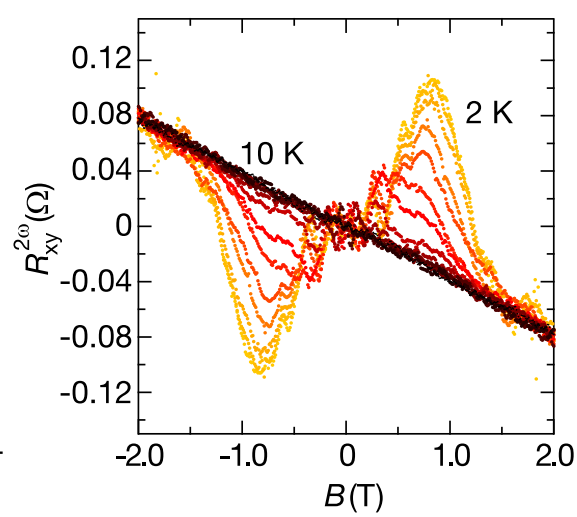

FIG. 6. Raw/antisymmetrized second-harmonic resistance and second-harmonic magnetoresistance in transversal direction in sample 2. (a) Raw and (b) antisymmetrized second-harmonic resistance as a function of magnetic field at $I=17 \mu \mathrm{A}$ and $T=2 \mathrm{~K}$ in sample 1 . (c) Temperature variations of $R_{x y}^{2 \omega}$ in sample 2 . The temperature-independent $B$-linear background persists above $T_{\mathrm{c}}=7.3 \mathrm{~K}$.

Considering a system in the normal state with broken inversion symmetry and magnetic field $B$, an electrical resistance $R$ is generally written as

$$
R=R_{0}(1+\gamma B I),
$$

where $R_{0}$ is a resistance under zero magnetic field and $\gamma$ the coefficient of nonreciprocal resistance in the normal state [18-20]. It is noted that the nonreciprocity emerges as a term in $R$ proportional to both $B$ and $I$.

However, when the nonreciprocal transport stems from vortex flow rectified by asymmetric pinning potentials [35], $R$ is expressed by

$$
R=R^{(1)}\left(1+\gamma^{\prime} \hat{B} I\right)
$$

where $R^{(1)}$ is the resistance of first order, $\gamma^{\prime}$ the coefficient of the nonreciprocal resistance in this case, and $\hat{B}=\frac{B}{|B|}$ the sign of magnetic field. It is noted that $R^{(1)}$ shows linearlike behavior but is even as a function of $B$. Therefore, in this formula, the nonreciprocal term in $R$ is also proportional to $B$ as well as $I$. This definition is reasonable because nonreciprocity in the vortex flow region is governed by the number of vortices, resulting in its linear dependence of $R^{(1)}$ on $B$. When the ac bias current (source-drain current) with a frequency of $\omega\left(I=I_{0} \cos \omega t\right)$ is applied, the output voltage is expressed as follows:

$$
V=R^{(1)} I_{0} \cos \omega t+\frac{R^{(1)} \gamma^{\prime} \hat{B} I_{0}^{2}}{2}(1+\cos 2 \omega t) .
$$

Both $\omega$ and $2 \omega$ components appear in Eq. (A3). At that time, by extracting the $\omega$ component $\left(R^{\omega}\right)$, we obtain

$$
R^{\omega}=R^{(1)} \text {. }
$$

On the other hand, by extracting the $2 \omega$ component $\left(R^{2 \omega}\right)$,

$$
R^{2 \omega}=\frac{1}{2} R^{\omega} \gamma^{\prime} \hat{B} I_{0}
$$

is obtained. Thus, to determine the $\gamma^{\prime}$ value, we defined $\gamma^{\prime}$ as

$$
\gamma^{\prime}=\frac{2 R^{2 \omega}}{R^{\omega} \hat{B} I_{0}} .
$$

We further approximated $R^{2 \omega}$ and $R^{\omega}$ in Eq. (A6) by the peak value $\left(R_{\text {peak }}^{2 \omega}\right)$ and the $R^{\omega}$ value at the peak position $\left(R_{\text {peak }}^{\omega}\right)$, respectively. This definition is different from one used in the previous study [23] (approximated as $\gamma=\frac{2 R_{\text {peak }}^{2 \omega}}{R_{\text {peak }}^{\omega} B_{\text {peak }} I_{0}}$, where the $B_{\text {peak }}$ is the magnetic field at the peak position). In the previous study, we thought of an effect of paraconductivity on nonreciprocal transport and thus expanded the concept of nonreciprocal transport in the normal state [18-20], where nonreciprocal resistance is proportional to both $B$ and $I$. However, in the present system, the contribution of not particles but vortices is dominant. Therefore, we use the definition of the $\gamma^{\prime}$ value shown above. We compare it with $\gamma$ used in a previous study in Figs. 5(c) and 5(d). The sudden increase in $\gamma$ just below $T_{\mathrm{c}}$ described by the paraconductivity effect does not exist in $\gamma^{\prime}$ because of the decrease in $B_{\text {peak }}$ as $T$ approaches $T_{\mathrm{c}}$. In the present case, i.e., the low temperature region, where $R^{\omega}$ and $R^{2 \omega}$ show linear dependence on $B$ because the characteristics of the system is governed by number of vortices, the definition of $\gamma^{\prime}$ might be more plausible.

\section{APPENDIX B: SYMMETRIZATION AND ANTISYMMETRIZATION}

To analyze the nonreciprocal transport, we symmetrized or antisymmetrized the raw data obtained in the present experiment. We measured the magnetoresistance for both positive and negative magnetic field $\left[R_{\exp }(B)\right]$ and extracted the odd or even component as a function of $B$ by the procedures shown below.

To extract symmetrized and antisymmetrized $\left[R_{\text {sym }}(B)\right.$ and $R_{\text {asym }}(B)$ ] resistance from the raw data, we calculated

$$
R_{\text {sym }}(B)=\frac{R_{\text {exp }}(B)+R_{\text {exp }}(-B)}{2}
$$

and

$$
R_{\text {asym }}(B)=\frac{R_{\text {exp }}(B)-R_{\text {exp }}(-B)}{2},
$$



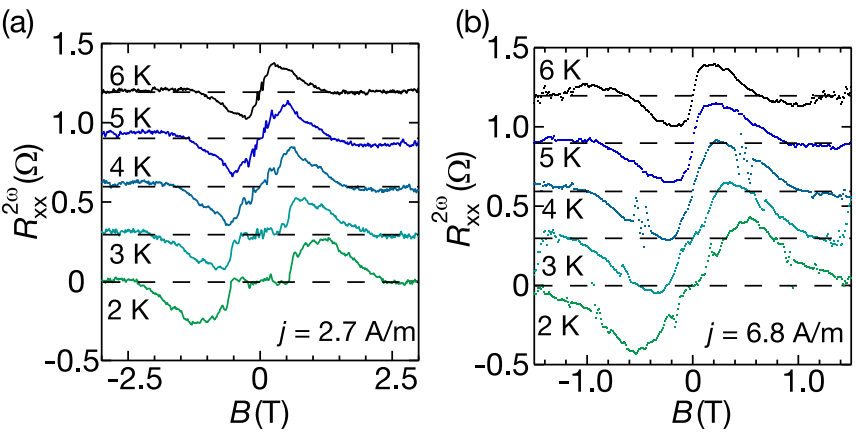

FIG. 7. Temperature variations of second-harmonic resistance. (a), (b) Second-harmonic magnetoresistance for temperatures varying in $1-\mathrm{K}$ steps from 2 to $6 \mathrm{~K}$ for $j=2.7$ (a) and $6.8 \mathrm{~A} / \mathrm{m}$ (b).

respectively. $R_{\text {sym }}(B)$ and $R_{\text {asym }}(B)$ are even and odd as a function of $B$, respectively. We adopt this symmetrization or antisymmetrization for $R^{\omega}$ and $R^{2 \omega}$ in the main text. We provide typical raw data and antisymmetrized data in Figs. 6(a) and $6(\mathrm{~b})$, respectively.

The linear background component observed in Fig. 2(e) can be attributed to the Hall effect originating from the nonlinear component of source-drain current $\left(I^{2 \omega}\right)$, namely, a component which is proportional to the square of input ac current in the current flowing through the sample. This $I^{2 \omega}$ possibly comes from geometrical asymmetry of source and drain electrodes in the sample because $B$-linear background persists even above $T_{\mathrm{c}}=7.3 \mathrm{~K}$ and has no temperature dependence as shown in Fig. 6(c). When an out-of-plane magnetic field is applied, $I^{2 \omega}$ generates the transverse second-harmonic resistance in a similar manner as the normal Hall effect in the first-harmonic component, which cannot be distinguished from the intrinsic nonreciprocal resistance in the normal state. However, the anomalous increase of the $2 \omega$ component with the peak in the superconducting states cannot be explained by the geometrical asymmetry, and thus we can safely conclude that this behavior intrinsically arises from the crystal symmetry of $\mathrm{MoS}_{2}$.

\section{APPENDIX C: SELECTION RULES WITH THREEFOLD ROTATIONAL SYMMETRY}

According to an argument of the point group symmetry, second-order response in the present trigonal system can be written as

$$
j_{x}^{(2)} \propto E_{x}^{2}-E_{y}^{2}
$$

and

$$
j_{y}^{(2)} \propto E_{x} E_{y},
$$

which are also described in the previous study [23]. In this formula, $j^{(2)}$ and $E$ are the second-harmonic current and the applied electric field, respectively, and the $x(y)$ direction corresponds to the zigzag (armchair) direction. Note that this argument is simply derived from the point group theory and can be applied also to the nonreciprocity originating from vortex motions discussed in the present study. The above

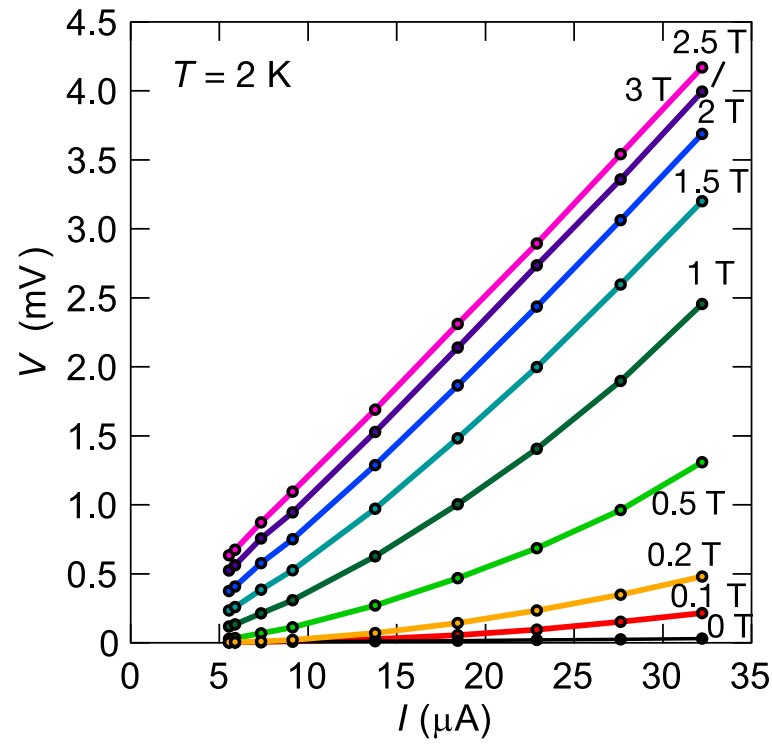

FIG. 8. $I-V$ curves at several magnetic fields. $I-V$ characteristics are measured at magnetic fields varying in $0.1-\mathrm{T}$ steps from 0 to $0.2 \mathrm{~T}$ and 0.5 -T steps from 0.5 to $3 \mathrm{~T}$.

directional dependence of the second-harmonic response indicates that nonreciprocal transport can be observed only along the longitudinal (transversal) direction when applied electric field or current is parallel to zigzag (armchair) direction (configurations $\mathrm{A} / \mathrm{B}$ ), which is consistent with our results.

\section{APPENDIX D: TEMPERATURE VARIATIONS OF SECOND-HARMONIC RESISTANCE}

In Figs. 7(a) and 7(b), we show temperature variations of second-harmonic magnetoresistance at $j=2.7$ [Fig. 7(a)] and $6.8 \mathrm{~A} / \mathrm{m}$ [Fig. 7(b)]. These $B-T$ planes are utilized to accomplish the comprehensive $B-j-T$ vortex phase diagram shown in Fig. 4(e). In the $B-T$ phase diagram observed at several $j$ values, the thermal creep state continuously evolves to the plastic vortex flow region with decreasing temperature. This $B-T$ phase diagram is smoothly connected to the dynamical $B-j$ plane at the lowest temperature.

\section{APPENDIX E: CURRENT-VOLTAGE (I-V) CHARACTERISTICS}

We show $I-V$ characteristics at several magnetic fields in Fig. 8. At low current states, the $I-V$ characteristics are highly nonlinear at low magnetic fields, whereas they tend to exhibit linear behavior above $0.2 \mathrm{~T}$. This linearity is consistent with the picture of the quantum metallic state. However, it is difficult to identify the boundary between the quantum metallic state and the plastic vortex flow state (or thermal creep state), which would come between 1 and $1.5 \mathrm{~T}$, from the $I-V$ characteristics. This is simply because the nonreciprocal component is on the order of $10^{-3}$ of the linear resistance $R_{x x}^{\omega}$. The second-harmonic resistance $R_{x x}^{2 \omega}$ is so sensitive that we are able to capture the nonreciprocity/vortex ratchet effect, which is not noticeable simply from the $I-V$ characteristics. 
[1] Y. Saito, T. Nojima, and Y. Iwasa, Highly crystalline 2D superconductors, Nat. Rev. Mater. 2, 16094 (2016).

[2] J. $\mathrm{Wu}$ and $\mathrm{H}$. Weitering, Focus on superconductivity in the 2D limit, Supercond. Sci. Technol. (2017), http://iopscience.iop.org/journal/0953-2048/page/Focuson-Superconductivity-in-the-2D-Limit.

[3] N. Reyren, S. Thiel, A. D. Caviglia, L. F. Kourkoutis, G. Hammerl, C. Richter, C. W. Schneider, T. Kopp, A. S. Ruetschi, D. Jaccard, M. Gabay, D. A. Muller, J. M. Triscone, and J. Mannhart, Superconducting interfaces between insulating oxides, Science 317, 1196 (2007).

[4] T. Zhang, P. Cheng, W. J. Li, Y. J. Sun, G. Wang, X. G. Zhu, K. He, L. Wang, X. Ma, X. Chen, Y. Wang, Y. Liu, H. Q. Lin, J. F. Jia, and Q. K. Xue, Superconductivity in one-atomic-layer metal films grown on Si(111), Nat. Phys. 6, 104 (2010).

[5] Q. Y. Wang, Z. Li, W. H. Zhang, Z. C. Zhang, J. S. Zhang, W. Li, H. Ding, Y. B. Ou, P. Deng, K. Chang, J. Wen, C. L. Song, K. He, J. F. Jia, S. H. Ji, Y. Y. Wang, L. L. Wang, X. Chen, X. C. Ma, and Q. K. Xue, Interface-induced high-temperature superconductivity in single unit-cell FeSe films on $\mathrm{SrTiO}_{3}$, Chin. Phys. Lett. 29, 037402 (2012).

[6] Y. Saito, Y. Kasahara, J. Ye, Y. Iwasa, and T. Nojima, Metallic ground state in an ion-gated two-dimensional superconductor, Science 350, 409 (2015).

[7] A. W. Tsen, B. Hunt, Y. D. Kim, Z. J. Yuan, S. Jia, R. J. Cava, J. Hone, P. Kim, C. R. Dean, and A. N. Pasupathy, Nature of the quantum metal in a two-dimensional crystalline superconductor, Nat. Phys. 12, 208 (2016).

[8] N. P. Breznay and A. Kapitulnik, Particle-hole symmetry reveals failed superconductivity in the metallic phase of twodimensional superconducting films, Sci. Adv. 3, e1700612 (2017).

[9] C. H. Sharma, A. P. Surendran, S. S. Varma, and M. Thalakulam, 2D superconductivity and vortex dynamics in 1T$\mathrm{MoS}_{2}$, Commun. Phys. 1, 90 (2018).

[10] M. P. A. Fisher, Quantum Phase Transitions in Disordered TwoDimensional Superconductors, Phys. Rev. Lett. 65, 923 (1990).

[11] A. M. Goldman, Superconductor-insulator transitions, Int. J. Mod. Phys. B 24, 4081 (2010).

[12] D. Ephron, A. Yazdani, A. Kapitulnik, and M. R. Beasley, Observation of Quantum Dissipation in the Vortex State of a Highly Disordered Superconducting Thin Film, Phys. Rev. Lett. 76, 1529 (1996)

[13] A. Kapitulnik, S. A. Kivelson, and B. Spivak, Anomalous metals: Failed superconductors, Rev. Mod. Phys. 91, 011002 (2019).

[14] I. Tamir, A. Benyamini, E. J. Telford, F. Gorniaczyk, A. Doron, T. Levinson, D. Wang, F. Gay, B. Sacépé, J. Hone, K. Watanabe, T. Taniguchi, C. R. Dean, A. N. Pasupathy, and D. Shahar, Sensitivity of the superconducting state in thin films, Sci. Adv. 5, eaau3826 (2019).

[15] E. Shimshoni, A. Auerbach, and A. Kapitulnik, Transport through Quantum Melts, Phys. Rev. Lett. 80, 3352 (1998).

[16] P. Phillips and D. Dalidovich, The elusive Bose metal, Science 302, 243 (2003).

[17] D. Das and S. Doniach, Bose metal: Gauge-field fluctuations and scaling for field-tuned quantum phase transitions, Phys. Rev. B 64, 134511 (2001).

[18] G. L. J. A. Rikken, J. Fölling, and P. Wyder, Electrical Magnetochiral Anisotropy, Phys. Rev. Lett. 87, 236602 (2001).
[19] G. L. J. A. Rikken and P. Wyder, Magnetoelectric Anisotropy in Diffusive Transport, Phys. Rev. Lett. 94, 016601 (2005).

[20] F. Pop, P. Auban-Senzier, E. Canadell, G. L. J. A. Rikken, and N. Avarvari, Electrical magnetochiral anisotropy in a bulk chiral molecular conductor, Nat. Commun. 5, 3757 (2014).

[21] T. Ideue, K. Hamamoto, S. Koshikawa, M. Ezawa, S. Shimizu, Y. Kaneko, Y. Tokura, N. Nagaosa, and Y. Iwasa, Bulk rectification effect in a polar semiconductor, Nat. Phys. 13, 578 (2017).

[22] F. Qin, W. Shi, T. Ideue, M. Yoshida, A. Zak, R. Tenne, T. Kikitsu, D. Inoue, D. Hashizume, and Y. Iwasa, Superconductivity in a chiral nanotube, Nat. Commun. 8, 14465 (2017).

[23] R. Wakatsuki, Y. Saito, S. Hoshino, Y. M. Itahashi, T. Ideue, M. Ezawa, Y. Iwasa, and N. Nagaosa, Nonreciprocal charge transport in noncentrosymmetric superconductors, Sci. Adv. 3, e1602390 (2017).

[24] Y. M. Itahashi, T. Ideue, Y. Saito, S. Shimizu, T. Ouchi, T. Nojima, and Y. Iwasa, Nonreciprocal transport in gate-induced polar superconductor $\mathrm{SrTiO}_{3}$, Sci. Adv. 6, eaay9120 (2020).

[25] R. H. Friend and A. D. Yoffe, Electronic properties of intercalation complexes of the transition metal dichalcogenides, Adv. Phys. 36, 1 (1987).

[26] Q. H. Wang, K. Kalantar-Zadeh, A. Kis, J. N. Coleman, and M. S. Strano, Electronics and optoelectronics of two-dimensional transition metal dichalcogenides, Nat. Nanotechnol. 7, 699 (2012).

[27] Y. Guo, C. Liu, Q. Yin, C. Wei, S. Lin, T. B. Hoffman, Y. Zhao, J. H. Edgar, Q. Chen, S. P. Lau, J. Dai, H. Yao, H. S. P. Wong, and Y. Chai, Distinctive in-plane cleavage behaviors of two-dimensional layered materials, ACS Nano 10, 8980 (2016).

[28] Y. Saito, T. Nojima, and Y. Iwasa, Quantum phase transitions in highly crystalline two-dimensional superconductors, Nat. Commun. 9, 778 (2018).

[29] M. V. Feigel'man, V. B. Geshkenbein, and A. I. Larkin, Pinning and creep in layered superconductors, Phys. C (Amsterdam, Neth.) 167, 177 (1990).

[30] Y. Saito, Y. Nakamura, M. S. Bahramy, Y. Kohama, J. Ye, Y. Kasahara, Y. Nakagawa, M. Onga, M. Tokunaga, T. Nojima, Y. Yanase, and Y. Iwasa, Superconductivity protected by spinvalley locking in ion-gated $\mathrm{MoS}_{2}$, Nat. Phys. 12, 144 (2016).

[31] J. M. Lu, O. Zeliuk, I. Leermakers, N. F. Q. Yuan, U. Zeitler, K. T. Law, and J. T. Ye, Evidence for two-dimensional Ising superconductivity in gated $\mathrm{MoS}_{2}$, Science 350, 1353 (2015).

[32] J. E. Villegas, S. Savel, F. Nori, and E. M. Gonzalez, A superconducting reversible rectifier that controls the motion of magnetic flux quanta, Science 302, 1188 (2003).

[33] J. E. Villegas, E. M. Gonzalez, M. P. Gonzalez, J. V. Anguita, and J. L. Vicent, Experimental ratchet effect in superconducting films with periodic arrays of asymmetric potentials, Phys. Rev. B 71, 024519 (2005).

[34] C. C. de Souza Silva, J. Van de Vondel, M. Morelle, and V. V. Moshchalkov, Controlled multiple reversals of a ratchet effect, Nature 440, 651 (2006).

[35] S. Hoshino, R. Wakatsuki, K. Hamamoto, and N. Nagaosa, Nonreciprocal charge transport in two-dimensional noncentrosymmetric superconductors, Phys. Rev. B 98, 054510 (2018).

[36] A. Kato and Y. Tanimura, Quantum suppression of ratchet rectification in a Brownian system driven by a biharmonic force, J. Phys. Chem. B 117, 13132 (2013).

[37] K. Hamamoto, T. Park, H. Ishizuka, and N. Nagaosa, Scaling theory of a quantum ratchet, Phys. Rev. B 99, 064307 (2019). 